\title{
"Es rico hacerlos, pero no tenerlos": análisis de la violencia obstétrica durante la atención del parto en Colombia ${ }^{1}$
}

\author{
"It's Nice to Make them but not Have Them": \\ Analysis of Obstetric Violence during Delivery \\ Care in Colombia
}

\section{"É bom faze-los, mas não os ter": análise da violência obstétrica durante a atenção do parto na Colômbia}

Viviana Valeria Vallana Sala, $\mathrm{MSc}^{2 *}$

Recibido: 12 de abril de 2018 - Aceptado: 20 de septiembre de 2018

Doi: http://dx.doi.org/10.12804/revistas.urosario.edu.co/revsalud/a.8125

Para citar este artículo: Vallana- Sala VV. "Es rico hacerlos, pero no tenerlos": análisis de la violencia obstétrica durante la atención del parto en Colombia. Rev Cienc Salud. 2019;17(especial):128-144. Doi: http://dx.doi.org/10.12804/revistas.urosario.edu.co/revsalud/a.8125

\section{Resumen}

Introducción: se analizan, desde una perspectiva feminista, algunas formas de maltrato y violación de los derechos humanos, sexuales y reproductivos de las mujeres durante la atención del embarazo y parto, fenómeno conocido como violencia obstétrica. Desarrollo: se analizan las historias de embarazo/parto de seis mujeres que parieron sus hijos/as en instituciones hospitalarias, tanto públicas como privadas, así como la autoetnografía de los dos embarazos/partos de la autora, para analizar las maneras en las que las mujeres describen y dan sentido a sus experiencias de violencia obstétrica durante la atención de su parto. En el artículo, se visibilizan las diversas formas de violencia que componen la violencia obstétrica, como institucional, física, verbal, psicológica y simbólica. Conclusiones: se aportan algunos elementos que, a juicio de las mujeres y de la Organización Mundial de la Salud, deben transformarse para garantizar un trato digno y el derecho a un parto y nacimiento en condiciones de respeto y seguridad.

Palabras clave: violencia obstétrica, embarazo, parto, derechos humanos, derechos sexuales y reproductivos, parto respetado.

Los testimonios (historias de embarazo y parto) que se utilizan en este artículo, además del ejercicio autoetnográfico de los dos embarazos/partos de la autora, hacen parte de la investigación realizada entre los años 2014 y 2016 para la realización de la tesis: "Parirás con dolor, lo embarazoso de la práctica obstétrica: discursos y prácticas que naturalizan la violencia obstétrica en Bogotá”, que se presentó para optar al título de magíster en Estudios Culturales de la Pontificia Universidad Javeriana de Bogotá, en 2016 (1).

1 Universidad del Rosario. Miembro de Huitaca: Observatorio de Salud Sexual y Reproductiva de Colombia.

Autora de correspondencia: valeria_vallana@hotmail.com 
"Es rico hacerlos, pero no tenerlos": análisis de la violencia obstétrica durante la atención del parto en Colombia

Abstract

Introduction: This article analyzes some forms of mistreatment and violation of the human, sexual and reproductive rights of women during the pregnancy, delivery and childbirth care, phenomenon known as obstetric violence. Development: It analyzes the pregnancy/childbirth histories of six women who gave birth their children in public and private hospitals, as well as the auto-ethnography of the two pregnancies/delivery of the author; to analyze the ways in which women describe and give meaning to their experiences of obstetric violence during their delivery care. In the article, the different forms of violence that make up obstetric violence are visualized, such as institutional, physical, verbal, psychological and symbolic violence. Conclusions: Analyzing the elements that, in the opinion of women and the World Health Organization, must be transformed to guarantee a dignified care and the right to a delivery and birth in conditions of respect and security.

Keywords: Obstetric violence, pregnancy, delivery, childbirth, human rights, sexual and reproductive rights, respected delivery.

\section{Resumo}

Introdução: analisam-se, desde uma perspectiva feminista, algumas formas de abuso e violação dos direitos humanos, sexuais e reprodutivos, das mulheres durante a atenção da gravidez e parto, fenômeno conhecido como violência obstétrica. Desenvolvimento: se analisam as histórias de gravidez/parto de seis mulheres que pariram aos seus filhos/as em instituições hospitalares tanto públicas quanto privadas, assim como a etnografia das duas gravidezes/partos da autora; para analisar as maneiras como as mulheres descrevem e são sentido às suas experiências de violência obstétrica durante a atenção de seu parto. No artigo, se visibilizam as diversas formas de violência que compõem a violência obstétrica, como institucional, física, verbal, psicológica e simbólica. Conclusões: aportam-se alguns elementos que, ao juízo das mulheres e a Organização Mundial da Saúde, devem transformar-se para garantir um tato digno e o direito a um parto e nascimento em condições de respeito e segurança.

Palavras-chave: violência obstétrica, gravidez, parto, direitos humanos, direitos sexuais e reprodutivos, parto respeitado.

\section{Introducción}

L $^{l}$ sistema de salud y sus profesionales son entendidos como quienes reparan el mal que 工 ocasiona la violencia, pero nunca como posibles perpetuadores de esta. En este sentido, es fundamental analizar las diversas maneras en que el ejercicio del poder, en el ámbito sanitario, desencadena diversos tipos de maltrato: violencia física, psicológica, verbal, simbólica, entre otras (1-4). Se trata de violencias estructurales encubiertas, invisibles o invisibilizadas, normalizadas y naturalizadas que, en su ejercicio, degradan tanto a quienes las ejercen, como a quienes la reciben.

Muchas veces, la jefe le dice a uno “déjela que grite un poco, ya se callará” y uno mismo dice esas cosas, les dice mamita, gordita, las manda callar cuando los gritos o el llanto lo desesperan a uno... pero créeme que cuando yo acompañé a mi hermana y vi cómo la trataban... eso me dolió a mí, pues porque es mi hermana y la quiero, eso me hizo darme cuenta de 
"Es rico hacerlos, pero no tenerlos": análisis de la violencia obstétrica durante la atención del parto en Colombia

muchas cosas, de lo que uno hace, a veces sin darse cuenta, del daño que está haciendo. (Relato de estudiante de enfermería, 2015 oct 05)

Como se puede deducir del testimonio anterior, muchas conductas aprendidas se reproducen, sin reparar en los efectos y daños que ocasionan en las mujeres que están siendo atendidas en las instituciones, por lo que es necesario hacer un cuestionamiento acerca del grado de conocimiento y naturalización que tiene el personal de la salud sobre las violencias que se pudieran estar reproduciendo en su práctica cotidiana; ya sea de manera consciente 0 inconsciente, voluntaria o involuntaria para, de esta manera, contribuir a visibilizar aquellos efectos adversos que pueden llegar a presentarse por este tipo de conductas, durante cualquier momento de la ruta de atención de las gestantes.

Por esta razón, en estas páginas se analizarán algunas de las percepciones de las mujeres, sobre las experiencias de violencia que tuvieron que afrontar durante sus contactos con el personal de la salud, en la atención de sus procesos fisiológicos de embarazo y parto; con la intención de visibilizar y contribuir a la desnaturalización de la violencia obstétrica y, de esta manera, proporcionar las bases para el entendimiento de aquellos comportamientos que se reproducen y hacen parte del quehacer cotidiano de la práctica profesional del personal de la salud, que pudieran estar violentando por acción u omisión a las pacientes.

Las historias de embarazo/parto de las mujeres, y los testimonios que hacen parte de esta investigación cualitativa-interpretativa, se fueron construyendo mediante varios encuentros y entrevistas a profundidad, donde seis mujeres relataban aquellos deseos, miedos y expectativas que tenían sobre la experiencia del parto antes y durante el embarazo, así como el recuerdo de su experiencia durante su atención. Se realizaron alrededor de tres o cuatro encuentros de dos a tres horas con cada una de las participantes, y cada una de ellas firmó un consentimiento informado en el que se estipulaba que accedía de manera voluntaria a participar en esta investigación, bajo la promesa de la autora de cambiar su nombre y resguardar su identidad. Cada una de las entrevistas fue grabada y transcrita para su posterior análisis y triangulación. Para la selección de la muestra, el único criterio consistió en que la conformaran mujeres que hubieran parido uno o varios de sus hijos en instituciones hospitalarias públicas o privadas del país.

En cuanto a la autoetnografía de los dos embarazos y partos de la autora, hace parte del ejercicio de reconocer la importancia de la experiencia personal de quien investiga, como lo indica Ana Sabrina Mora:

Con las técnicas de recolección de la información con las que contamos, [...] es más sencillo conocer las prácticas por medio de las observaciones y comprender las representaciones mediante los discursos, que las experiencias del cuerpo [...]. Una vez concluyamos que además del trabajo de campo tradicional se necesita la inclusión del conocimiento corporeizado producido desde la experiencia personal, surgen nuevas preguntas. (5) 
"Es rico hacerlos, pero no tenerlos": análisis de la violencia obstétrica durante la atención del parto en Colombia

Este tipo de antropología encarnada le otorga centralidad a la experiencia corporal de los/las investigadores, lo que permite conocer a profundidad las sensaciones corporales como insumo en la investigación (6).

La autoetnografía me permitió reconocer e identificar las sensaciones, frustraciones, miedos, resistencias, y facilitó el entendimiento y la identificación de las experiencias de violencia que sufrimos las mujeres en la atención de nuestros procesos.

Si bien no siempre el personal de la salud involucrado en la atención a las maternas está dispuesto a reconocer la existencia de la violencia obstétrica, es vital recordar los movimientos que, alrededor del mundo, claman por la humanización del parto o, por lo menos, por el derecho de las mujeres a acceder a una atención respetuosa y segura del parto/nacimiento ${ }^{3}$. Particularmente, en el país está la reciente emergencia de dos observatorios concentrados en especial en la visibilización de este tipo de violencias: el Observatorio de Violencia Obstétrica de Colombia y Huitaca: Observatorio de Salud Sexual y Reproductiva de Colombia, ambas iniciativas que surgieron en 2016 con el fin de hacer un seguimiento a la garantía de los derechos sexuales y reproductivos de las mujeres en la atención de sus procesos.

Aunque pareciera una exageración la proclama de exigir respeto para las mujeres en estos contextos, incluso la oms en su comunicado "Prevención y erradicación de la falta de respeto y el maltrato durante la atención del parto en centros de salud” declara:

Muchas mujeres sufren un trato irrespetuoso y ofensivo durante el parto en centros de salud, que no solo viola los derechos de las mujeres a una atención respetuosa, sino que también amenaza sus derechos a la vida, la salud, la integridad física y la no discriminación. Esta declaración reclama un accionar más enérgico, diálogo, investigación y apoyo en relación con este importante problema de salud pública y de derechos humanos. (7)

Ante este panorama, es de vital importancia, como primer paso para la transformación, el reconocimiento de que la problemática existe y que, tal como lo sugiere la oms, se debe promover la investigación multidisciplinar y socializar sus resultados, para brindarle al personal de la salud las herramientas necesarias para reconocer aquellas prácticas violentas que ha normalizado (7).

Así mismo, es importante que se considere el implemento y la inclusión de las medidas que recomienda la oms en las Guías de Atención del Parto Normal que desde 1996 se vienen actualizando, como la guía "Recomendaciones de la oms para la Conducción del Trabajo de Parto" y las más recientes del 15 de febrero de 2019: Individualized, Supportive Care Key to Positive Childbirth Experience, Says wно (8, 9). En este reciente comunicado, se incluyen 56 recomendaciones basadas en la evidencia científica, que propenden por partos/nacimientos seguros, atendidos bajo el

3 Véase, por ejemplo, el trabajo de colectivos como Las Casildas en Argentina (http://www.lascasildas.com.ar/); El parto es nuestro, en España (https://www.elpartoesnuestro.es/), presente también, en México y Ecuador y Los Observatorios de Violencia Obstétrica en Argentina, Chile, Brasil y Francia.

Rev. Cienc. Salud. Bogotá, Colombia, vol.17 (2): 128-144, julio de 2019 
"Es rico hacerlos, pero no tenerlos": análisis de la violencia obstétrica durante la atención del parto en Colombia

principio del respeto de los derechos de las mujeres y sus hijos/as. Entre las nuevas recomendaciones, se hace un llamado de atención sobre las intervenciones rutinarias y protocolarias que se están empleando en mujeres que no las requieren, afectando negativamente la experiencia del parto/nacimiento. Se insta a los profesionales e instituciones a respetar los tiempos del proceso fisiológico del parto, a adaptar las medidas de atención a las particularidades de cada parto y no adaptar el parto a las necesidades institucionales o personales del personal de la salud; a facilitar y garantizar el acompañamiento de una persona de la elección de la mujer, así como al respeto y trato en condiciones de dignidad, que le permitan a la mujer decidir activamente sobre la experiencia y la atención que desea recibir, entre otras. Se recomienda especialmente poner en el centro de la atención a la mujer y sus necesidades y atender, no solamente al resultado del parto/nacimiento, sino también a la calidad de la experiencia.

El esfuerzo por la implementación de estas recomendaciones en Colombia beneficiaría a las mujeres y su experiencia durante la atención del parto, ya que en el país se reproducen los modelos que autoras como Davis-Floyd y Gutman, definen como modelos tecnocráticos e industrializados del parir/nacer $(10,11)$.

El modelo tecnocrático de parir/nacer se caracteriza por elementos que han alcanzado un alto grado de normalización en la atención de los partos hospitalarios en el país, incluso los partos de bajo riesgo. Algunos de sus paradigmas y características son la consideración de las maternas como ‘objetos’ de intervención, más que como sujetos de derecho y de decisión; el entendimiento del cuerpo gestante como cuerpo funcional, que debe cumplir con unos requisitos previamente estandarizados; la estandarización de protocolos, donde todas las mujeres son sometidas a los mismos procedimientos e intervenciones protocolarias, aun cuando no los necesiten; la fragmentación mente-cuerpo, característica del paradigma biomédico, donde se concentran las medidas en los órganos y su funcionamiento, desligándose de las características identitarias y socioculturales de las gestantes; contexto altamente jerarquizado, donde las maternas ocupan una posición de subordinación, por ello son alienadas de su rol activo durante el parto, otorgando notoriedad, protagonismo y poder autoritativo al personal de la salud que las atiende; lógicas neoliberales donde las instituciones buscan ganancias económicas y reducción de gastos y, por último, una muy alta sobrevaloración de la tecnología aplicada al embarazo/parto, como los resultados de ecografías y monitoreos, que desplazan las relaciones intersubjetivas de las pacientes con el personal de la salud (10).

Como se puede notar, este modelo guarda una estrecha relación con los modelos industriales automatizados, donde se optimiza el tiempo por medio de las cadenas estandarizadas de producción, por lo que también se ha llamado el modelo industrializado de parir/nacer o modelo de producción de la reproducción $(1,11)$. Laura Gutman describe la experiencia de parto industrializado de esta manera: 
"Es rico hacerlos, pero no tenerlos": análisis de la violencia obstétrica durante la atención del parto en Colombia

Me refiero al nacimiento industrializado, vivido tanto desde el lugar de la parturienta como desde el lugar del bebé: ambos manipulados, cortados, separados, pinchados, abandonados, violentados, medicados, anestesiados, monitoreados, vigilados y filmados [...], el sufrimiento se hace presente cuando los partos son conducidos y las mujeres somos infantilizadas, maltratadas, pinchadas, cortadas, abandonadas humilladas y tratadas como un cuerpo funcional que tiene la obligación de responder al resultado esperado. Aparece confusamente cuando somos acostadas en posición dorsal (boca arriba) con los pies atados y los genitales al descubierto, recibiendo órdenes incomprensibles y amenazas sobre el tiempo de descuento antes de ser castigadas con una cesárea. Así, el sufrimiento puede ser insoportable, aunque la anestesia borre todo dolor físico. (11)

Consecuencia de este modelo y de las variadas formas de violencia que se reproducen en su implementación, surge el concepto de violencia obstétrica, inicialmente desde la terminología legal de países como Venezuela, Argentina y México, entre otros, donde las leyes en cuanto a la violencia de género incluyen y sancionan aquellas violencias que afectan a las mujeres durante la atención en salud, con especial énfasis en el proceso y la atención del parto hospitalario. El concepto ha sido también utilizado desde varias organizaciones feministas como los Observatorios de Violencia Obstétrica y de Salud Sexual y Reproductiva que, desde la sociedad civil y en varios países, abogan para visibilizar las violencias encubiertas que resultan del contacto de las mujeres con el personal de la salud, en la atención autoritaria de sus procesos sexuales y reproductivos.

Así mismo, varios trabajos desarrollados desde las ciencias sociales han adoptado el término violencia obstétrica o violencia ginecobstétrica, por su capacidad tanto explicativa como política, al mostrar las diversas formas de violencia que se presentan en la atención de varios procesos sexuales y reproductivos de las mujeres, como la anticoncepción, las consultas en ginecología y la atención obstétrica en embarazo, parto, aborto y puerperio (1-4, 12-18). Esto, asociado con el poder obstétrico que deviene del conocimiento autoritativo que históricamente se ha asociado con la biomedicina para el manejo de los procesos de salud/ enfermedad y del ciclo vital de las mujeres $(12,14)$. Lo que da como resultado un ejercicio del poder que viola los derechos humanos, sexuales y reproductivos de las mujeres cuando se realiza de manera autoritaria $(13,15)$.

Para el análisis contextual del fenómeno, en Colombia se cuenta con escasas investigaciones que, desde los Estudios de Género los Estudios Culturales y la Enfermería, analizan la problemática, sin que logren impactar e incidir en las prácticas de la atención biomédica del proceso de parto/nacimiento del país (1-3, 19).

Por ello, a continuación, se hará una contextualización de la emergencia del concepto de violencia obstétrica y se mostrarán algunas de las formas en las que se ejerce en el país, para concluir en las recomendaciones que podrían ayudar a su visibilización y erradicación. 
"Es rico hacerlos, pero no tenerlos": análisis de la violencia obstétrica durante la atención del parto en Colombia

\section{Desarrollo}

$\mathrm{V}$

enezuela fue el primer país en cuya legislación se menciona la violencia obstétrica, en la Ley Orgánica Sobre el Derecho de las Mujeres a una Vida Libre de Violencia, que entró en vigencia el 19 de marzo de 2007. En esta ley se define violencia obstétrica como

La apropiación del cuerpo y los procesos reproductivos de las mujeres por el personal de la salud, que se expresa en un trato deshumanizador, en un abuso de medicalización y patologización de los procesos naturales, trayendo consigo pérdida de autonomía y capacidad de decidir libremente sobre sus cuerpos y sexualidad, impactando negativamente en la calidad de vida de las mujeres. (20)

En el artículo 15, numeral 13, se establecen sanciones a las instituciones y al personal de la salud que incurra en alguna de las siguientes acciones:

1) No atender oportuna y eficazmente las emergencias obstétricas; 2) Obligar a la mujer a parir en posición supina y con las piernas levantadas, existiendo los medios necesarios para la realización de un parto vertical; 3) Obstaculizar el apego precoz del niño o niña con su madre, sin causa médica justificada, negándole la posibilidad de cargarlo o cargarla y amamantarlo o amamantarla inmediatamente al nacer; 4) Alterar el proceso natural del parto de bajo riesgo, mediante el uso de técnicas de aceleración, sin obtener el consentimiento voluntario, expreso e informado de la mujer; 5) Practicar el parto por vía cesárea, existiendo condiciones para el parto natural, sin obtener el consentimiento voluntario, expreso e informado de la mujer. (20)

Para el caso de Argentina, está la Ley de Protección Integral de las Mujeres (21). La Ley 26485, sancionada el 11 de marzo de 2009, en el artículo 6, inciso “e” define:

Violencia obstétrica: es aquella que ejerce el personal de salud sobre el cuerpo y los procesos reproductivos de las mujeres, expresada en un trato deshumanizado, un abuso de medicalización y patologización de los procesos naturales, de conformidad con la Ley 25929. (Inciso e): Se considera trato deshumanizado el trato cruel, deshonroso, descalificador, humillante o amenazante ejercido por el personal de salud en el contexto de la atención del embarazo, parto y postparto, ya sea a la mujer o al/la recién nacido/a, así como en la atención de complicaciones de abortos naturales o provocados, sean punibles o no. (21)

Desde el 30 de abril de 2014, México se suma a la visibilización y tipificación de la violencia obstétrica, definiéndola como:

La negligencia en la atención médica expresada en un trato deshumanizado; el abuso de medicalización o patologización de los procesos naturales, llegando incluso a tratarlos como si fueran emergencias obstétricas; la práctica abusiva de cesáreas pese a existir condiciones para el parto natural; el uso de métodos anticonceptivos o de la esterilización sin el consentimiento informado y voluntario de la madre, obstaculizar sin causa médica 
"Es rico hacerlos, pero no tenerlos": análisis de la violencia obstétrica durante la atención del parto en Colombia

justificada el apego precoz del recién nacido con su madre, negándole la posibilidad de acogerlo y amamantarlo inmediatamente al nacer, entre otros. (22)

Como se puede constatar en las definiciones legales del fenómeno, la violencia obstétrica está fuertemente ligada al ejercicio del poder y la autoridad, así como a las características de los modelos industrializados y tecnocráticos de parir/nacer antes mencionados (10,11).

La apropiación de los procesos fisiológicos reproductivos de las mujeres, por parte de las instituciones y el personal de la salud en el momento de la atención, tiene como resultado una pérdida de autonomía y poder de decisión de las mujeres, lo que las reduce a un rol pasivo que impacta negativamente en la experiencia de procesos como el parto. Todas estas lógicas se derivan del principio de medicalización/patologización de los procesos fisiológicos femeninos - menstruación, embarazo, aborto, parto, puerperio y menopausia—que, convertidos en asuntos de intervención médica, sitúan a las mujeres en una posición de subordinación ante los regímenes de saber-poder de la biomedicina y sus practicantes (23-27). Estos regímenes de saber-poder, implantados históricamente de manera autoritaria sobre las formas de conocimiento y representación que las mujeres hacían de sus procesos fisiológicos, han resultado en una violencia epistémica, consistente en la anulación y subvaloración de todos aquellos conocimientos y saberes empíricos de las mujeres sobre sus procesos y experiencias, en favor de conocimientos extrañados con el peso de la legitimidad y la autoridad de los discursos científicos de la biomedicina (28).

Concomitantemente, la violencia obstétrica es el resultado de una doble posición de subordinación de las mujeres gestantes y parturientas en los servicios de salud: 1) aquella que se deriva de la relación propia de su género en un sistema patriarcal, lo que la hace una violencia de género y estructural, y 2) la posición de subordinación que tienen los/las pacientes en un medio altamente jerarquizado, como lo es el sector de la salud; por esta razón, la violencia obstétrica es el resultado de la intersección entre violencia de género y violencia institucional en la atención en salud $(1,29)$. Esta doble posición de subordinación se complejiza cuando se trata de mujeres racializadas, indígenas, negras o de niveles socioeconómicos bajos, en este sentido, análisis interseccionales sobre raza, clase y género, y las diferencias en cuanto a la atención en salud y la calidad del servicio que se presta a las mujeres en condiciones de marginalidad y vulnerabilidad, son fundamentales para descubrir las complejas relaciones de poder y dominación que se materializan en violencias con muchas y, a veces, muy graves consecuencias en la salud física y emocional de estas mujeres y sus hijos o hijas. Tras esta contextualización y definición del concepto de violencia obstétrica, es importante analizar aquellas violencias que la componen. 
"Es rico hacerlos, pero no tenerlos": análisis de la violencia obstétrica durante la atención del parto en Colombia

\section{Violencia simbólica}

La violencia simbólica es el resultado de la interiorización y la naturalización de la relación de subordinación y dependencia de las mujeres dentro del sistema de atención en salud. Por ello, existe una suerte de aceptación de las propias afectadas, que hace que las mujeres justifiquen, excusen e incluso agradezcan el ejercicio de la dominación y la violencia que recae sobre ellas $(1-4,15)$. "Es así como las mujeres invisibilizan situaciones e interacciones donde han sido maltratadas en alguna forma, e incluso llegan a justificar esas prácticas por su propia actitud. Al introyectar la culpa, justifican los castigos que pueden recibir” (4). La violencia simbólica contribuye, en gran medida, a la naturalización e invisibilización de las mujeres de las prácticas violentas que recaen sobre ellas.

Pues a mí me dolía mucho entonces sí, me decían que el doctor, el de la anestesia no había llegado, que estaba durmiendo y que había que dejarlo descansar, o algo así. Pero yo no aguantaba más, entonces sí estaba como muy intensa y llamaba a las enfermeras todo el tiempo. Yo creo que ellas también ya estaban desesperadas y por eso era que no me trataban tan bien. (Relato de María, 2015 may 19)

\section{Violencia institucional}

La violencia institucional en violencia obstétrica se caracteriza por protocolizar la atención de las gestantes dentro de las instituciones hospitalarias (1-4). La realización de intervenciones médicas protocolarias sin justificación médica violenta, tanto a la mujer que es intervenida sin necesitarlo ni autorizarlo, como al personal de la salud que se ve obligado a su cumplimiento, aun cuando su experiencia y criterio sean contrarios a esa normatividad.

[...] en el curso me dijeron que me comprara un cojín de esos que es como un flotador, con un hueco en el centro. Cuando otra señora les preguntó para qué era, le contestaron que para la herida de la episiotomía. Me acuerdo que después de que la enfermera explicó para que se hacía, otra señora dijo que a ella, cuando le preguntara el médico, le iba a decir que a ella no se lo hiciera, que ella no quería, pero la vieja le contestó: “mamita, eso no se pregunta, el médico le dice le voy a cortar y la corta, él no le va a preguntar si usted quiere o no, solamente le informa, o no, simplemente lo hace y ya. (Relato de Virginia, fecha 2015 mar 15)

En el curso psicoprofiláctico nos estaban explicando los cuidados que hay que tener con la herida de la episiotomía, sobre el dolor y el ardor que era normal sentir y sobre la importancia de la dieta para no tener estreñimiento [...] la enfermera decía: "si no me hacen caso con lo de la comida y les da estreñimiento van a ver las estrellas de dolor". Entonces otra señora preguntó: "pero esa episiotomía solamente se hace si es necesario, ¿cierto?” Y la enfermera le contestó en tono como de burla: "no mamá, todas acá van a salir con episiotomía, de eso ninguna se salva, a menos de que le toque una cesárea, en ese caso los cuidados de la herida quirúrgica son los mismos. (Relato de Paloma, 2015 may 20) 
"Es rico hacerlos, pero no tenerlos": análisis de la violencia obstétrica durante la atención del parto en Colombia

Como se puede ver en estos relatos sobre la preparación al parto de Virginia y Paloma, las intervenciones médicas protocolarias se dan a conocer desde el embarazo, ya sea en las consultas médicas o en los cursos psicoprofilácticos, naturalizándolas y haciéndolas parecer intervenciones necesarias y, sobre todo, obligatorias (1-2). La imposición autoritaria y sin capacidad de réplica de las intervenciones son ejemplo de esa apropiación de la experiencia del parto, así como de la anulación de los deseos y decisiones de las mujeres a la hora de decidir el tipo de atención e intervenciones que consienten en su parto.

A mí no me preguntaron: “te podemos hacer” nada, la hicieron y ya. De hecho, hasta después me enteré porque sentí como que me estaban, pues dormida y todo, pero sentí que estaban cosiéndome. O sea, no me dijeron si se podía, no se podía.... De hecho, me enteré después porque tenía la herida y los puntos, pero yo no sabía que le hacían a uno eso, nadie me lo dijo. (Relato de María, 2015 may 19)

Como se ve, también hay procedimientos protocolarios que se practican sobre el cuerpo de las gestantes sin que ellas sean informadas o consultadas, lo que se traduce un ejercicio autoritario de la intervención médica $(1,2)$.

Existen elementos simbólicos que facilitan el ejercicio de esa violencia institucional, como la conversión simbólica de las gestantes en pacientes, siendo ataviadas con las mismas indumentarias y nominadas todas bajo el apelativo de "mamá”, “madre”, “mamita”, “gorda”, "gordita”. Esta homogenización simbólica facilita la repetición de protocolos institucionales y universaliza el tipo de atención requerida, sin atender a la particularidad de cada caso (1-4).

\section{Violencia física}

Junto con la violencia institucional están las modalidades de violencia física de la violencia obstétrica. Una de ellas está asociada precisamente con la repetición de protocolos en una mujer que no requiera de intervenciones durante su parto. En este sentido, toda intervención realizada en una gestante, que no esté médicamente justificada y aprobada por la mujer, puede ser considerada como violencia obstétrica. De la misma manera, forzar a una mujer a consentir intervenciones como cesáreas, por medio del uso y abuso del poder médico obstétrico, por medio de la administración del miedo por el estado de vulnerabilidad, constituye una violación de sus derechos humanos (1, 12, 30, 31).

Así mismo, la violencia física también se expresa en intervenciones médicas dolorosas, realizadas sin utilización de alguna medida que mitigue el dolor, existiendo los medios y las condiciones para utilizar analgesia o anestesia; los tactos vaginales reiterados y realizados por más de una persona y la realización de maniobras e intervenciones de manera brusca y poco considerada con la mujer que las recibe.

[...] Como el señor me estaba haciendo daño, entonces yo me tensioné. Entonces el señor dijo que me tenía que relajar. Yo intentaba relajarme, pero el señor me volvía a hacer 
"Es rico hacerlos, pero no tenerlos": análisis de la violencia obstétrica durante la atención del parto en Colombia

daño, entonces al final el señor se puso pues grosero conmigo y me empezó a decir que me relajara que no le estaba dejando hacer su trabajo. Entonces yo le dije: "que usted me está haciendo daño” y el señor volvió a meter los dedos con fuerza, volvió a hacerme daño y bueno la cosa terminó en que yo terminé callada llorando porque el tipo fue bastante miserable. (Relato de Virginia, 2015 mar 15)

En este relato, se pone de manifiesto el comportamiento que el personal de la salud espera de las pacientes, “que soporten el dolor”, “que colaboren” y dejen trabajar al personal de la salud; de lo contrario, la paciente será tildada de "paciente difícil”, posición que autoriza a los profesionales a brindar un trato diferencial, prevenido y poco empático $(1,32)$.

También se considera violencia física la imposición de posturas, tanto durante el trabajo de parto como en el parto, así como cualquier medida que impida su movimiento, como la sujeción de las piernas durante el parto o las manos en la cesárea.

Cuando me mostraron a mi hijo, yo quería tocarlo, abrazarlo y movía los brazos y las manos para alcanzarlo, pero no podía porque estaba amarrada... eso es muy angustiante, ver que se lo están llevando sin tú siquiera haber podido tocarlo. Mira, uno quiere tocarlos verlos, acariciarlos, estar desnudos y abrazados y uno ahí amarrado a esa camilla, de verdad, porque son así, deberían dárselos a uno mientras lo cosen a uno, y revisar a los bebés encima de uno... no sé Valeria, eso pienso yo. (Relato de la autora)

\section{Violencia psicológica}

La violencia psicológica es el resultado de un trato deshumanizado que viola los derechos humanos, sexuales y reproductivos de las mujeres durante la atención (1-4,12-15, 25).

En este punto, es importante analizar la conceptualización de la mujer dentro de los modelos tecnocrático e industrializado del parir/nacer de las instituciones. Las mujeres en estos contextos son conceptualizadas como contaminantes para la atención cómoda del parto por parte del personal de la salud (4). En este sentido, la gestante, sus miedos, dudas, sensaciones y opiniones son invisibilizadas y suprimidas en tanto se percibe que contaminan, obstruyen, incomodan la labor del personal de la salud en el cumplimiento de los protocolos de atención (1). Así, las mujeres manifiestan haber sido invitadas en múltiples ocasiones durante su trabajo de parto a guardar silencio, “a colaborar” y a obedecer.

Sumado a esto, se encuentra la ausencia de respeto por aspectos relacionados con la intimidad de las mujeres ya que, en el ámbito hospitalario, el pudor y la vergüenza ante la exhibición de sus cuerpos y genitales desnudos no es tenida en consideración (1-4,13-14).

[...] uno es como un pedazo de carne ahí tirado, no les importa lo que uno sienta, si tiene pena, si se siente mal, a ellos solo les preocupan las cosas médicas. (Relato de Paloma, 2015 my 20) 
"Es rico hacerlos, pero no tenerlos": análisis de la violencia obstétrica durante la atención del parto en Colombia

Pues entraron... no sé cuántos, pero además me lo hacían diferentes personas. Entraban y “plum” y me hacían el tacto, entraban y "plum” me hacían otro tacto. [...] una de esas, vino un señor y me hizo el tacto [risas], y fue cuando salió el agua y luego no sabían si había roto fuentes o no había roto fuente, bueno un enredo. Luego ya uno estaba como de turno y se fue, y entonces vino otro, o sea eso fue toque y toque [...]. Todo el mundo metiéndole el dedo y mirando, y es que además no era uno, sino varios los que entraban ahí: “cómo va” y levantaban la sábana y lo miraban a uno y uno pues..., se sentía uno como... no sé, no era agradable. (Relato de Virginia, 2015 mar 15)

Así mismo, las formas de violencia verbal hacen parte de la violencia psicológica, cuando, por ejemplo, el personal de la salud regaña, se burla o insulta a las pacientes o, se hace alusión a la sexualidad de las mujeres, o al evento placentero sexual que desencadena el embarazo y el parto $(1-4,13-14)$.

Pocas veces en la vida había sentido tanto dolor, ese tacto fue muy doloroso, ¡tenaz! No te imaginas el dolor, entonces pues yo grité “aaaahh” y me dice el médico como: "pero me imagino que cuando se la metió el que la embarazó ahí si no gritaba”. (Relato de Susana, 2015 abr 08)

La enfermera me decía constantemente: “es que es rico hacerlos, pero no tenerlos”. (Relato de la autora)

[...] yo me porté bien, no me quejé nada, es que yo no quería que me trataran como a otra señora que estaba allí, que se quejaba mucho de dolor y gritaba mucho [...] si viera como la trataban, eso le decían "cállese vieja tal por cual, ya nos tiene mamados con esa gritadera, eso sí quien la manda, no haberse puesto en esas, si quiere que le demos algo entonces cállese”, incluso un médico le dijo: “¿acaso me llamó a pedirme ayuda cuando estaba haciendo al muchachito?” entonces, como yo ya había visto eso, cuando me empezó a doler mucho, pues me aguanté todo lo que podía para que me trataran bien, ¿si me entiende? (Relato de Rosa)

Yo escuchaba cómo las mujeres gritaban de dolor pidiendo que les ayudaran y escuchaba a una enfermera que las mandaba a callar de una manera agresiva, les decía que solamente había un anestesiólogo y que todavía no era su turno. Había una que pedía ayuda a gritos decía: "me duele mucho, por favor ayúdenme, denme algo para el dolor, no puedo más” ¡tenaz, pobrecita! la enfermera le decía, así en plan de regaño, como: "cállese, no es nuestra culpa, va a asustar a las demás, si no se calla la meto en un cuarto a usted sola”. (Relato de Virginia, 2015 nov 11)

En estos relatos se ponen de manifiesto varios tipos de violencia, y es interesante encontrar la manera en la que la mención de la culpa es un poderoso dispositivo de encausamiento de las conductas de las mujeres que están siendo atendidas. La culpa y el castigo se hacen visibles ante la actitud, muchas veces punitiva, que asume el personal de la salud, en estos 
"Es rico hacerlos, pero no tenerlos": análisis de la violencia obstétrica durante la atención del parto en Colombia

casos bajo la amenaza del aislamiento, el buen trato, o la demora en la administración de anestésicos (1-2, 4).

Seguir órdenes y soportar regaños hacen parte también de este tipo de violencia, y son recordados de manera vívida por las mujeres en su experiencia de parto hospitalario.

“Acuéstese”, “levántese”, “abra las piernas”, “relájese”, “puje”, “respire”. "Lista, ahora puje”, “no, así no, puje duro, eso, así, puje, puje, puje, uno, dos tres cuatro, pare, descanse, respire... otra vez, puje uno, dos, tres, ya viene, listo, ya salió la cabeza, ahora no vaya a pujar, no puje, cuando yo le diga... algo así es como lo recuerdo”. (Relato de María, 2015 may 19)

Vea, a uno le dicen como que "a usted todavía le falta mucho, no vaya a pujar, respire como le enseñaron, ¿si fue al curso?”, y yo tenía muchas ganas de pujar... y cuando sentí era que el bebé ya estaba saliendo. Entonces yo grite y vino una y me regañó que por no haber avisado antes, que por haberme puesto a pujar sin que me dijeran, que se podía haber caído el niño de la camilla, que yo tan irresponsable. (Relato de Rosa, 2015 nov 11)

Todos los anteriores ejemplos de conducta del personal de la salud y las instituciones en la atención de los partos constituyen una violación de los derechos de las mujeres; lo que impacta negativamente en la experiencia de sus partos e incrementa el riesgo de efectos adversos en su salud tanto física como emocional.

\section{Conclusiones}

— $\mathrm{n}$ estas páginas, se ha analizado el contexto de emergencia del concepto de violencia L obstétrica y la manera como este ha adquirido relevancia, no solo desde el ámbito legal donde nace, sino también en las ciencias sociales que analizan las relaciones de poder dentro del ámbito médico, y sus efectos en la vida y la salud de las mujeres en la experiencia de procesos como el parto.

Con el objetivo de atender la problemática, es importante visibilizar algunas de las expectativas que tienen las mujeres que han experimentado violencia obstétrica en la atención de sus partos. Estas recomendaciones concuerdan con los preceptos del parto respetado, y las sugerencias de la oms para la atención de los partos/nacimientos en condiciones de seguridad y dignidad.

Las mujeres instan a instituciones y profesionales a respetar las decisiones, la autonomía, la privacidad, la intimidad y los deseos de la dueña del parto como sujeto de derecho. Entender y obrar en consecuencia de la fisiología del parto, de que cada parto es diferente y, por ello, las medidas de atención deben acomodarse y adaptarse más a quien está pariendo, y menos a los requerimientos institucionales y profesionales. Facilitar y garantizar el vínculo temprano madre/hijo/a inmediatamente tras el parto/nacimiento, si las condiciones de 
"Es rico hacerlos, pero no tenerlos": análisis de la violencia obstétrica durante la atención del parto en Colombia

salud de madre o hijo/a son adecuadas, inclusive durante la cesárea. Permitir y garantizar el movimiento y las posturas que desee asumir la dueña del parto; procurar durante el embarazo una información veraz, clara y suficiente que le permita tomar decisiones informadas durante el parto; consultar y solicitar el permiso de la dueña del parto ante cada examen e intervención; facilitar el acompañamiento de una persona de la elección de la mujer, respetar sus tiempos, espacios, miedos, pudor e intimidad. Evitar cualquier referencia o juicio de índole moral que pueda intervenir en el trato ofrecido a la dueña del parto, ya sea por edad, clase social, cantidad de hijo/as, creencias, origen étnico, estado civil, etc. (1) En una frase: las mujeres exigen respeto.

Por estas razones, se insta a las diferentes instituciones hospitalarias y al Ministerio de Salud, a implementar las recomendaciones de la oms para la atención del parto, para, de esta manera, reducir la violencia institucional inmersa dentro de la violencia obstétrica. Esta implementación mitigaría los efectos adversos y las violencias que los modelos tecnocráticos e industrializados de parir/nacer, hegemónicos en el país, tienen sobre las experiencias de parto de las mujeres que paren y sus hijo/as que nacen. De esta manera, se recomienda a los profesionales de la salud e instituciones, no solamente atender al buen resultado de un parto/nacimiento, sino también a la experiencia vital de las mujeres y sus hijo/as durante la vivencia de estos procesos. En este sentido, es de vital importancia el entendimiento de las mujeres parturientas como dueñas de sus partos y sujetos de derecho para decidir sobre el tipo de atención e intervenciones que desean recibir $(1,33)$.

Para la transformación del modelo actual, hacia un modelo respetado de atención, es fundamental también la dignificación de las condiciones laborales del personal de la salud, atender a sus horarios y honorarios; así como a las violencias institucionales que recaen sobre ello/as en el ejercicio de su profesión. En este sentido, las temáticas abordadas en este artículo deben hacer parte de la formación de los profesionales de la salud, para proporcionar las herramientas que les permitan una comunicación asertiva con los/las pacientes, problematizando las violencias que resultan de las relaciones de poder y dominación.

Así mismo, el modelo de información/educación de las gestantes debe propender hacia la socialización de información veraz, clara y suficiente, que le permita tomar decisiones como sujeto de derechos, evitando el modelo actual de naturalización de las intervenciones e imposición de estas.

Por otra parte, se invita a los profesionales de la salud a reflexionar sobre su posición de poder en la atención de personas en estado de vulnerabilidad, a la reflexividad crítica sobre su accionar profesional y el nivel de impacto que, por acción u omisión, tienen sobre una experiencia vital, como lo es el parto/nacimiento. En este sentido, el artículo invita al sector de la salud a hacer sus saberes más contestables, a permitir la discusión interdisciplinar que contribuya a mejorar y dignificar, tanto su ejercicio profesional, como la atención que reciben las mujeres. 
"Es rico hacerlos, pero no tenerlos": análisis de la violencia obstétrica durante la atención del parto en Colombia

\title{
Descargos de responsabilidad
}

\author{
T a autora de este trabajo asume la responsabilidad de lo publicado, y la Revista de Ciencias \\ U de la Salud no asume ninguna responsabilidad por esto.
}

\section{Referencias}

1. Vallana V. Parirás con dolor, lo embarazoso de la práctica obstétrica. Discursos y prácticas que naturalizan la violencia obstétrica en Bogotá. Bogotá: Pontificia Universidad Javeriana; 2016.

2. Monroy S. El continuo ginecobs-tétrico: Experiencias de violencia vividas por mujeres gestantes en servicios de salud en Bogotá. Bogotá: Universidad Nacional de Colombia; 2012.

3. Restrepo A, Rodríguez D, Torres N. “Me des-cuidaron el parto” La violencia obstétrica y el cuidado recibido por el personal de la salud a mujeres durante su proceso de parto. Bogotá: Pontificia Universidad Javeriana; 2016.

4. Sadler M. Así me nacieron a mi hija. Aportes antropológicos para el análisis de la atención biomédica del parto hospitalario. Santiago: Universidad de Chile; 2003.

5. Mora AS. El Cuerpo investigador el cuerpo investigado. Una aproximación fenomenológica a la experiencia del puerperio. Revista Colombiana de Antropología, 2009;45(I)11-37. Disponible en: http://www.scielo.org.co/scielo.php?script=sci_abstract\&pid=S0486-65252009000100002\&lng=en\&nrm=iso\&tlng=es

6. Esteban ML. Antropología encarnada. Antropología desde una misma. Papeles del cEIc 2004;(12):1-21.

7. Organización Mundial de la Salud. Prevención y Erradicación de la Falta de Respeto y el Maltrato Durante la Atención del Parto en Centros de Salud [internet] 2014 [citado 2018 abr 05]. Disponible en: http://apps.who.int/iris/bitstream/handle/10665/134590/ WHO_RHR_14.23_spa.pdf?sequence=1

8. Organización Mundial de la Salud. Recomendaciones de la oms para la conducción del Trabajo de Parto [internet] 2015 [citado 2018 abr 09]. Disponible en: http://www.who. int/topics/maternal_health/directrices_OMS_parto_es.pdf

9. Organización Mundial de la Salud. Individualized, Supportive Care Key to Positive Childbirth Experience, Says wHo [on line]. 2018 [cited 2018 Apr 07]. Disponible en: http:// www.who.int/mediacentre/news/releases/2018/positive-childbirth-experience/en/\#

10. Davis-Floyd R. "The technocratic model of birth.” En: S Tower Hollis, L Pershing, MJ Young editors, Feminist Theory in the Study of Folklore. Illinois: U. of Illinois Press; 1993. p. 297-326.

11. Gutman L. Puerperios y otras exploraciones del alma femenina. Buenos Aires: Editorial del Nuevo Extremo; 2007.

12. Arguedas G. La violencia obstétrica: propuesta conceptual a partir de la experiencia Costarricense. En Cuadernos Inter.c.a.mbio sobre Centroamérica y el Caribe, 2014;11(1):145-69. Doi: 10.15517/C.A..V11I1.14238 
"Es rico hacerlos, pero no tenerlos": análisis de la violencia obstétrica durante la atención del parto en Colombia

13. Belli L. La violencia obstétrica: otra forma de violación de los derechos humanos. Unesco, Revista Redbioética, 2013;1(7):25-34.

14. Camacaro M. Patologizando lo natural, naturalizando lo patológico... improntas de la práxis obstétrica. Revista Venezolana de Estudios de la Mujer, 2009;14 (32):147-62. Disponible en: http://www.scielo.org.ve/scielo.php?script=sci_arttext\&pid=S1316-37012009000100011\&lng=es\&nrm=iso>. ISSN 1316-3701

15. Castro R, Erveti J. Sociología de la práctica médica autoritaria. Violencia obstétrica, anticoncepción inducida y derechos reproductivos. Cuernavaca: Universidad Nacional Autónoma de México; 2015.

16. Montes M. Las culturas del nacimiento: Representaciones y prácticas de las mujeres gestantes, comadronas y médicos. Tarragona: Universidad Rovira i Virgili; 2007.

17. Camacaro M. Voces sobre la violencia en la atención del aborto. Revista Venezolana de Estudios de la Mujer. 2006;11(26):55-70. Disponible en: http://www.scielo.org.ve/scielo. php?script=sci_arttext\&pid=S1316-37012006000100004\&lng=es\&nrm=iso>. ISSN 13163701

18. Tamayo J, Restrepo C, Gil L, González A. Violencia obstétrica y aborto. Aportes para el debate en Colombia. Bogotá: Grupo Médico por el Derecho a Decidir [internet]. 2015 [citado 2018 may 17]. Disponible en: http://www.safeabortionwomensright.org/wp-content/uploads/2016/02/GDC_Obstetric-Violence_ES-1.pdf

19. Colón C. Sentimientos, memorias y experiencias de las mujeres en trabajo de parto: El caso de centros hospitalarios en Cartagena. Cartagena: Universidad de Cartagena; 2008.

20. Venezuela. Asamblea Nacional. República Bolivariana de Venezuela. Ley Orgánica Sobre el Derecho de las Mujeres a una Vida Libre de Violencia (2007 abr 23).

21. Argentina. Senado y Cámara de Diputados de la Nación Argentina. Ley de Protección Integral de las Mujeres. Ley 26.485. (2009 abr01).

22. El Parto es Nuestro. México tipifica la violencia obstétrica [internet]. 2014 [citado 2018 abr 10]. Disponible en: https://www.elpartoesnuestro.es/blog/2014/06/16/mexico-tipifica-la-violencia-obstetrica

23. Ehrenreich B, English D. Por tu Propio Bien: 150 años de consejos expertos a mujeres. Madrid: Editorial Capitán Swing; 2010.

24. Valls-Llobet C. Mujeres, salud y Poder. Madrid: Ediciones Cátedra; 2009.

25. Bellón S. La violencia obstétrica desde los aportes de la crítica feminista y la biopolítica. Dilemata. 2015;1-7(18):93-111.

26. Tovar P. El Cuerpo subordinado y politizado: reflexión crítica sobre género y antropología médica. Revista Colombiana de Antropología, 2004;40:253-82.

27. Foucault M. Vigilar y castigar. Nacimiento de la prisión. Siglo xxi: Buenos Aires; 2002.

28. Viveros M. Saberes y dolores secretos. Mujeres salud e identidad. En: LG Arango, M León; M Viveros editoras, Género e Identidad. Ensayos sobre lo femenino y lo masculino. Bogotá: Ediciones Uniandes; 1995. p. 149-68.

29. Magnone N. Derechos sexuales y reproductivos en tensión: intervencionismo y violencia obstétrica. Trabajo presentado en las x Jornadas de Investigación de la Facultad 
"Es rico hacerlos, pero no tenerlos": análisis de la violencia obstétrica durante la atención del parto en Colombia

de Ciencias Sociales. 2011 sep 13-14; UdelaR, Montevideo [internet]. [citado 2018 may 16]. Disponible en: http://cienciassociales.edu.uy/wp-content/uploads/2013/archivos/ Magnone.pdf

30. Cáceres F, Nieves G. Atención Humanizada del Parto. Diferencial según condición clínica y social de la materna. Revista Colombiana de Obstetricia y Ginecología, 2017;68(2):12834. Doi: http://dx.doi.org/10.18597/rcog.3022

31. Diaz-Tello F. Invisible wounds: Obstetric violence in the United States. Reproductive Health Matters, 2016;24 (47):56-64. Doi: 10.1016/j.rhm.2016.04.004

32. Strauss A, Fagerhaugh S, Suczek B, Wiener C. El trabajo que realizan los pacientes hospitalizados. En: C de la Cuesta editora, Salud y enfermedad: Lecturas básicas en sociología de la medicina. Medellín: Editorial Universidad de Antioquia; 1999. p: 248-75.

33. Imaz E. Mujeres gestantes, madres en gestación. Metáforas de un cuerpo fronterizo. Universidad del País Vasco Política y Sociedad, 2001;(36):97-111. Doi: http://dx.doi. org/10.5209/POSO.25549 